2011

\title{
Theory and simulations of the scaling of magnetic reconnection with symmetric shear flow
}

\author{
P. A. Cassak
}

Follow this and additional works at: https://researchrepository.wvu.edu/faculty_publications

\section{Digital Commons Citation}

Cassak, P. A., "Theory and simulations of the scaling of magnetic reconnection with symmetric shear flow" (2011). Faculty Scholarship. 392.

https://researchrepository.wvu.edu/faculty_publications/392 


\title{
Theory and simulations of the scaling of magnetic reconnection with symmetric shear flow
}

\author{
P. A. Cassak ${ }^{\text {a) }}$ \\ Department of Physics, West Virginia University, Morgantown, West Virginia 26506, USA
}

(Received 20 March 2011; accepted 3 June 2011; published online 18 July 2011)

\begin{abstract}
The scaling of magnetic reconnection in the presence of an oppositely directed sub-Alfvénic shear flow parallel to the reconnecting magnetic field is studied using analytical scaling arguments and two-dimensional two-fluid numerical simulations of collisionless (Hall) reconnection. Previous studies noted that the reconnection rate falls and the current sheet tilts with increasing flow speed, but no quantitative theory was presented. This study presents a physical model of the effect of shear flow on reconnection, resulting in expressions for the scaling of properties such as the reconnection rate, outflow speed, and thickness and length of the dissipation region, which are verified numerically. Differences between Hall and Sweet-Parker reconnection are pointed out. The tilting of the current sheet is explained physically and a quantitative prediction is presented and verified. (C) 2011 American Institute of Physics. [doi:10.1063/1.3602859]
\end{abstract}

\section{INTRODUCTION}

In recent years, the study of magnetic reconnection has gone beyond the original studies ${ }^{1,2}$ that assumed a high degree of symmetry to make the problem more tractable. The breakdown of perfect symmetries involves asymmetries in magnetic field strength and density ${ }^{3,4}$ and three-dimensional effects. ${ }^{5}$ The present work addresses the effect of a shear flow, a bulk flow parallel to the reconnecting magnetic field. There will generally be some shear flow in any physical system; an example with significant shear flow is in tokamaks. ${ }^{6}$ Another common setting is at the dayside magnetopause.

Shear flow in magnetospheric reconnection is caused by the solar wind, and predominantly occurs at high latitudes, especially when the interplanetary magnetic field (IMF) points northward. The seminal observations ${ }^{7,8}$ revealed subAlfvénic shear flows at the dusk magnetopause. Reconnection influenced by shear flow is now routinely observed. ${ }^{9-18}$ Statistical studies showed that reconnection at the polar cusp occurs $90 \%$ of the time when the IMF is northward, ${ }^{19}$ and the flow speed during reconnection is sub-Alfvénic. ${ }^{20,61,62}$

There have also been many theoretical studies on the effect of shear flow. Early analytical studies addressed the effect of shear flow on the shock structure of reconnection. ${ }^{4,21,23}$ Shear flow in an unmagnetized plasma gives rise to the Kelvin-Helmoltz instability; it is stabilized by a magnetic field in the direction of the flow. This has prompted many studies on the linear theory of the Kelvin-Helmholtz and tearing instabilities in systems where both are present (e.g., Ref. 24). As the present study is on the nonlinear phase, we summarize only the studies most relevant for the present purposes. It was shown using magnetohydrodynamics (MHD) that reconnection is suppressed completely when the shear flow is greater than the Alfvén speed. ${ }^{21}$ However, both can be linearly unstable for the same parameters in Hall-MHD, ${ }^{25}$ which occurs because ions decouple from

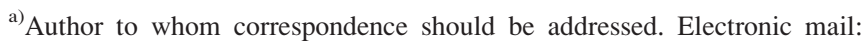
Paul.Cassak@mail.wvu.edu.
}

electrons near the current layer and the electron layer is tearing unstable while the ion layer is Kelvin-Helmholtz unstable.

The first large-scale numerical simulations ${ }^{26}$ used twodimensional MHD with a localized resistivity. They confirmed that reconnection does not occur if the flow is super-Alfvénic. (Interestingly, there is an analogy between this and the fact that reconnection is suppressed when the diamagnetic drift is super-Alfvénic, ${ }^{27}$ which has been confirmed by solar wind observations. ${ }^{28}$ ) When the flow is sub-Alfvénic, the dissipation region gets twisted and opens wider. A follow-up paper ${ }^{29}$ included the effects of an asymmetric density in the equilibrium, showing that the two effects can compete or enhance each other depending on parameters.

More recently, it was shown that the reconnection rate decreases with increasing shear flow speed, ${ }^{30,31}$ though no scaling law was presented. The nature and location of discontinuities was studied ${ }^{32}$ using hybrid simulations. Microand macro-instabilities were studied using particle-in-cell (PIC) simulations. ${ }^{33}$ Hall-MHD simulations were used to show that shear flow causes vortices in magnetic islands. ${ }^{34}$ Recent studies considered the effect of varying thicknesses of the shear flow layer using $\mathrm{PIC}^{35}$ and $\mathrm{MHD}^{31}$ simulations. Particle-in-cell simulations addressed the combined effects of asymmetries, a guide field, and shear flow. ${ }^{36}$ Related studies include signatures of flux transfer events, ${ }^{37}$ the behavior of current sheets in a unidirectional shear flow, ${ }^{38-41}$ and the effect of shear flow on secondary islands. ${ }^{42}$ The effect of shear flows on secondary Buneman instabilities was recently studied with PIC simulations. ${ }^{63}$

The goal of the present work is to understand quantitatively how reconnection scales as a function of shear flow. In addition to being useful for interpreting satellite data, this may be important for applications to solar wind-magnetospheric coupling. Borovsky ${ }^{43}$ recently proposed that the coupling is strongly dependent on parameters at the local reconnection site rather than the solar wind. A coupling function was derived, and the correlation between solar wind data and geomagnetic indices is very good. The model did not include shear flow effects, so this could be one way to improve the model. 
A theoretical model of the scaling of reconnection with shear flow is presented in Sec. II. Two-fluid simulations of Hall reconnection are compared to the theory in Sec. III. Results are discussed in Sec. IV. The present study concerns only the nonlinear phase of reconnection and does not treat Kelvin-Helmholtz unstable systems.

\section{THEORY}

In this section, a scaling analysis of reconnection with a symmetric shear flow parallel to the reconnecting magnetic field is presented. The same assumptions made in the SweetParker analysis ${ }^{1}$ are made here: Reconnection is two-dimensional with no initial out-of-plane (guide) magnetic field and has reached a steady state. For simplicity, the present analysis omits asymmetries in quantities such as magnetic fields, densities, temperatures, and shear flow speeds on either side of the dissipation region. With an eye toward space and laboratory applications, collisionless (Hall) reconnection is considered here; the effect of shear flow on Sweet-Parker (collisional) reconnection is discussed briefly in Sec. III A.

A schematic diagram of the dissipation region is in Fig. 1. The upstream magnetic field has strength $B$, the plasma mass density is $\rho$, the outflow speed is $v_{\text {out }}$, and the magnitude of the shear flow is $v_{s}$. The half-thickness of the dissipation region is $\delta$ and the half-length is $L$.

The main effect of shear flow on reconnection is to make the driving of the outflow jets less efficient. The outflow jet is generated by the tension in newly reconnected field lines. When a shear flow is present, a newly reconnected field line finds itself immersed in a plasma with a bulk flow, which releases some of the tension in the field, decreasing the outflow speed. To quantify this, one expects from energetics

$$
\frac{1}{2} \rho v_{\text {out }}^{2} \sim \frac{B^{2}}{8 \pi}-\frac{1}{2} \rho v_{s}^{2} .
$$

Solving for $v_{\text {out }}$ gives

$$
v_{\text {out }} \sim \sqrt{c_{A}^{2}-v_{s}^{2}}=c_{A} \sqrt{1-\frac{v_{s}^{2}}{c_{A}^{2}}},
$$

where $c_{A}=B /(4 \pi \rho)^{1 / 2}$ is the Alfvén speed based on the reconnecting magnetic field $B$. Clearly, this expression

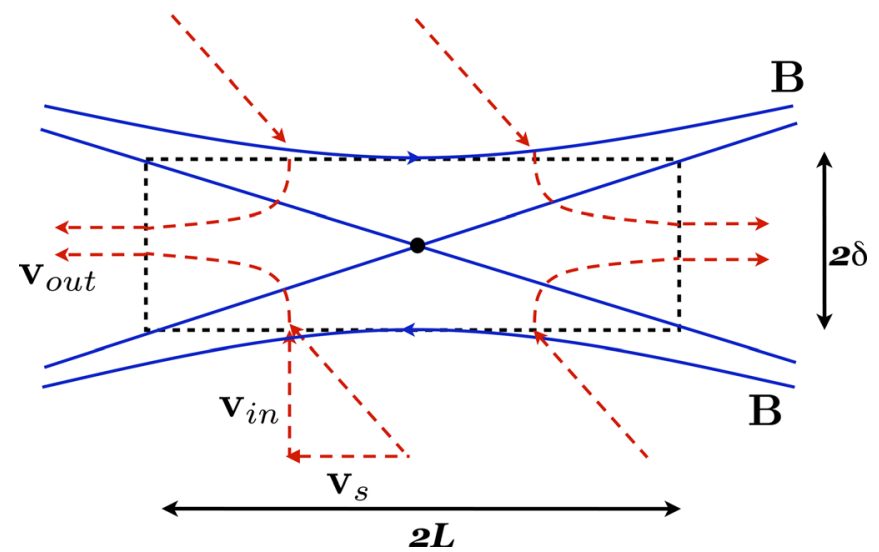

FIG. 1. (Color online) Drawing of the dissipation region in the presence of a shear flow. Magnetic field lines are (blue) solid lines, velocity flow lines are (red) dashed lines, and the edge of the dissipation region is the dashed (black) box. Reprinted from Ref. 44. reduces to the known result $v_{\text {out }} \sim c_{A}$ in the limit of zero shear flow. It is also consistent with reconnection shutting off for super-Alfvénic shear flows. Interestingly, this same multiplicative factor enters the linear theory of the tearing mode with a shear flow, although the full expression of the growth rate has a more complicated dependence on $v_{s}{ }^{24} \mathrm{It}$ is important to note that $B$ (and therefore $c_{A}$ ) may be a function of $v_{s}$, and in fact will be shown later that this is the case.

In collisionless reconnection, the outflow speed is intimately related to the thickness of the dissipation region. To see this, note that an ion entering the dissipation region is accelerated out of the plane by the reconnection electric field $E$. In a time $\Delta t$, it acquires an out-of-plane velocity $\Delta v_{z}$ of

$$
\Delta v_{z} \sim \frac{q E}{m} \Delta t
$$

where $q$ and $m$ are the ion charge and mass, respectively. The ions are deflected in the outflow direction by the reconnected magnetic field, so $\Delta v_{z} \sim v_{\text {out }}$. Now, $E$ is given by the reconnection rate $E \sim v_{\text {in }} B / c \sim v_{\text {out }} B \delta / L c$, where $v_{\text {in }}$ is the inflow speed and $v_{\text {in }} \sim v_{\text {out }} \delta / L$ from continuity. Since the time spent in the dissipation region is $\Delta t \sim L / v_{\text {out }}$, it follows from Eq. (2) that

$$
\delta \sim \frac{v_{\text {out }}}{\Omega_{c i}}
$$

where $\Omega_{c i}=q B / m c$ is the ion cyclotron frequency. This is similar to an argument used in Ref. 45. In the absence of shear flow, $v_{\text {out }} \sim c_{A}$, so $\delta \sim d_{i}=c / \omega_{p i}$, the ion inertial length, which is the expected result. Using Eq. (1) gives

$$
\delta \sim d_{i} \sqrt{1-\frac{v_{s}^{2}}{c_{A}^{2}}}
$$

Thus, the thickness of the layer decreases as a result of the shear flow.

The scaling of the length $L$ of the layer follows from a similar argument. Since the ions are redirected by the reconnected magnetic field, $L$ is given by the Larmor radius in the reconnected field

$$
L \sim \frac{v_{\text {out }}}{\Omega_{y}}
$$

where $\Omega_{y}=q B_{y} / m_{i} c$ is the cyclotron frequency based on the reconnected magnetic field $B_{y}$. Again using Eq. (1), one gets

$$
L \sim \frac{c_{A}}{\Omega_{y}} \sqrt{1-\frac{v_{s}^{2}}{c_{A}^{2}}}
$$

The scaling of the inflow speed follows from continuity. Using $v_{\text {in }} \sim v_{\text {out }} \delta / L$ and Eqs. (1), (3), and (4) gives

$$
v_{i n} \sim v_{i n, 0} \sqrt{1-\frac{v_{s}^{2}}{c_{A}^{2}}},
$$

where $v_{i n, 0}=c_{A y}=B_{y} /(4 \pi \rho)^{1 / 2}$ is the inflow speed in the absence of shear flow.

There is an interesting ramification of the thickness of the dissipation region decreasing. The magnetic field asymptotes 
to a value $B_{0}$, but it decreases at the dissipation region as it goes through zero at the center. When the dissipation region is thinner, it sees a smaller magnetic field upstream of the layer. Since the field changes approximately linearly with distance from the neutral line, one expects from Eq. (3) that

$$
B \sim B_{0} \sqrt{1-\frac{v_{s}^{2}}{c_{A}^{2}}}
$$

From this analysis, the scaling of the reconnection rate $E$ immediately follows. Since $E \sim v_{i n} B / c$, Eqs. (5) and (6) imply

$$
E \sim E_{0}\left(1-\frac{v_{s}^{2}}{c_{A}^{2}}\right)
$$

where $E_{0}=v_{i n, 0} B_{0} / c$ is the reconnection rate in the absence of shear flow. Note that Eqs. (6) and (7) were confirmed in Ref. 44.

The result in Eq. (6) allows for predictions for the outflow speed and length, solely in terms of $v_{s}$. Equations (1) and (4) become

$$
\begin{gathered}
v_{\text {out }} \sim c_{A 0}\left(1-\frac{v_{s}^{2}}{c_{A}^{2}}\right) \\
L \sim L_{0}\left(1-\frac{v_{s}^{2}}{c_{A}^{2}}\right),
\end{gathered}
$$

where $c_{A 0}=B_{0} /(4 \pi \rho)^{1 / 2}$ and $L_{0}=c_{A 0} / \Omega_{y}$ are the outflow speed and layer length in the absence of shear flow, and $B_{y}$ is assumed to be independent of $v_{s}$. The scaling expressions for $v_{i n}$ and $\delta$ are not affected by Eq. (6).

While the analysis here treats the ions, the arguments used to develop the scaling predictions should be similar for the electrons. Thus, it is expected that

$$
\begin{gathered}
B_{e} \sim B_{e 0} \sqrt{1-\frac{v_{s}^{2}}{c_{A}^{2}},} \\
v_{e, \text { out }} \sim c_{A e 0}\left(1-\frac{v_{s}^{2}}{c_{A}^{2}}\right), \\
\delta_{e} \sim d_{e} \sqrt{1-\frac{v_{s}^{2}}{c_{A}^{2}}}, \\
L_{e} \sim L_{e 0}\left(1-\frac{v_{s}^{2}}{c_{A}^{2}}\right),
\end{gathered}
$$

where the $e$ subscripts denote quantities referring to the electrons.

In addition to these quantities which typically specify the reconnection site, it has been previously observed that a shear flow causes the outflow jet and current sheet to tilt in the inflow direction towards the side in which the shear flow and the outflow are in opposite directions. ${ }^{26}$ The author knows of no quantitative prediction of this effect, so one is presented here.

In the absence of shear flow, outflow is generated by the tension force in newly reconnected magnetic field lines, as sketched in Fig. 2 on the left. One can think of a block (sketched in gray) as a fluid element attached to the field line

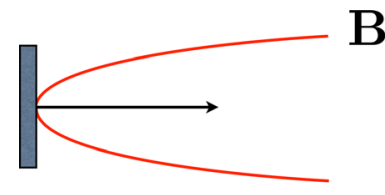

no shear flow

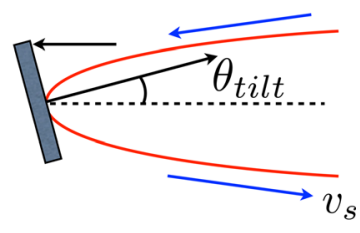

with shear flow
FIG. 2. (Color online) Sketches motivating why shear flow tilts the dissipation region. (a) Tension-driven outflow in the absence of shear flow and (b) the effect of dynamic pressure due to shear flow which tilts the jet in the direction of incoming shear flow, as observed in Ref. 26.

that drives the flow. With no shear flow, the outflow jet goes straight out to the right. In the presence of a shear flow, sketched on the right, the block feels a torque due to the dynamic pressure of the shear flow. In the sketch, the pressure gradient force is to the left on the top and right on the bottom. This twists the box up, so the outflow is driven toward the direction in which the outflow and shear flow are anti-aligned, which agrees with previous observations.

To make this quantitative, the tangent of the tilt angle $\theta_{\text {tilt }}$ (defined as the angle from the horizontal to the current sheet) is given simply by the ratio of the dynamic pressure $(1 / 2) \rho v_{s}^{2}$ to the force density due to the magnetic tension $B^{2} / 4 \pi$

$$
\tan \left(\theta_{\text {tilt }}\right) \sim \frac{(1 / 2) \rho v_{s}^{2}}{B^{2} / 4 \pi} \sim \frac{v_{s}^{2}}{2 c_{A}^{2}}
$$

The expressions derived here are tested with numerical simulations described in Sec. III.

\section{SIMULATIONS OF HALL RECONNECTION WITH SHEAR FLOW}

The scaling laws derived in the previous section are tested with two-dimensional simulations of compressible Hall reconnection with a shear flow using the two-fluid code F3D. ${ }^{46}$ Density, ion velocity, magnetic field, and pressure are evolved explicitly using the trapezoidal leapfrog in time and fourth order finite difference in space. Magnetic field strengths, number densities, velocities, lengths, electric fields, and pressures are normalized to $B^{\prime}, n^{\prime}$, the Alfvén speed $c_{A}^{\prime}=B^{\prime}$ $\left(4 \pi m n^{\prime}\right)^{1 / 2}$, the ion inertial length $d_{i}^{\prime}=\left(m c^{2} / 4 \pi n^{\prime} e^{2}\right)^{1 / 2}$, $E^{\prime}=c_{A}^{\prime} B^{\prime} / c$, and $P^{\prime}=B^{\prime 2} / 4 \pi$, respectively. The $x, y$, and $z$ directions are aligned with the initial directions of the magnetic field, the inflow, and the out-of-plane current.

The computational domain has a size $L_{x} \times L_{y}$ $=204.8 \times 102.4$ with a cell size of $0.05 \times 0.05$ and periodic boundary conditions are employed. The initial magnetic field profile is a double tearing mode configuration

$$
B_{x}(y)=\tanh \left(\frac{y-L_{y} / 4}{w_{0}}\right)-\tanh \left(\frac{y-L_{y} / 4}{w_{0}}\right)-1,
$$

where $w_{0}=1.0$ is the initial current sheet thickness. There is no initial out-of-plane (guide) magnetic field. The initial shear flow profile is

$$
v_{x}(y)=v_{s}\left[\tanh \left(\frac{y-L_{y} / 4}{w_{0}}\right)-\tanh \left(\frac{y-L_{y} / 4}{w_{0}}\right)-1\right],
$$



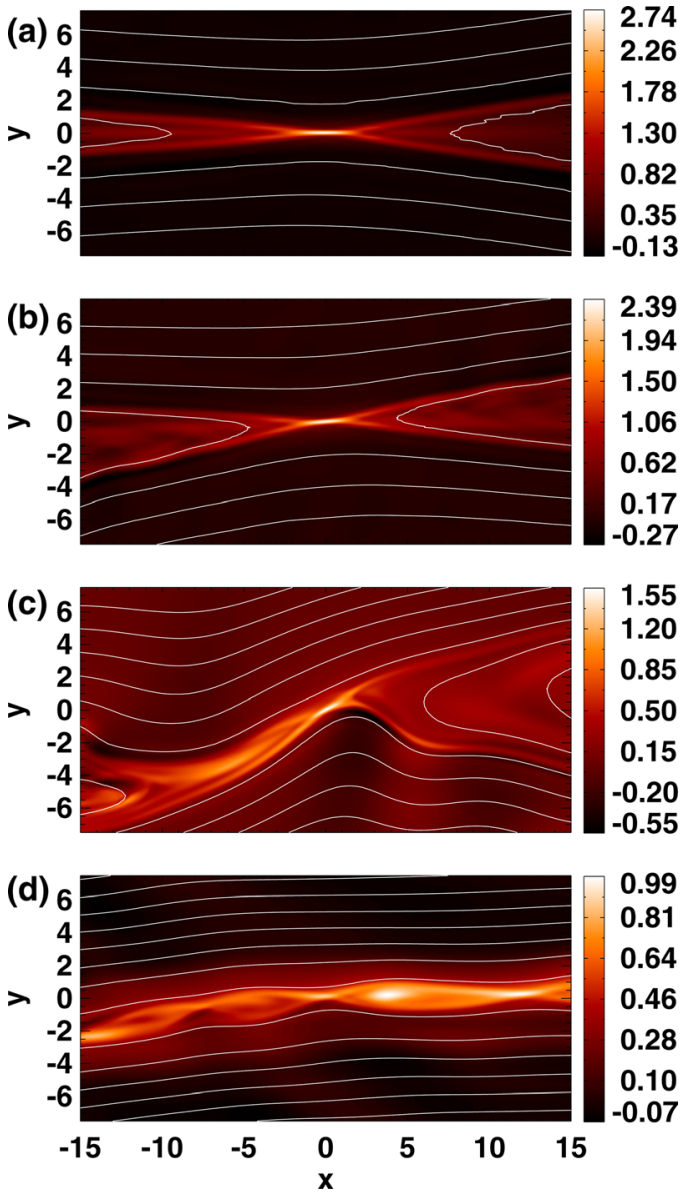

FIG. 3. (Color online) Out-of-plane current density $J_{z}$ (grayscale) with magnetic field lines (white lines) for simulations with $v_{s}$ of (a) 0.0 , (b) 0.4 , (c) 0.8 , and (d) $1.2 c_{A}$. Only a small portion of the computational domain is plotted. Shear flow and magnetic fields are to the left above and right below the current sheet.

with the same $w_{0}$ (although this is not necessary ${ }^{31}$ ). The initial density profile is uniform with $n=1$. The temperature has an asymptotic value of 1 far from the current sheet, but is initially non-uniform to ensure the initial configuration is an equilibrium.

The ratio of specific heats $\gamma$ is $5 / 3$. There is no viscosity or resistivity, but fourth order diffusion with coefficient $5 \times 10^{-5}$ is used in all the equations to damp noise at the grid scale. Reconnection is initiated by a magnetic perturbation $\delta \mathbf{B}=-\left(0012 B^{\prime} L_{y} / 2 \pi\right) \hat{\mathbf{z}} \times \nabla\left[\sin \left(2 \pi x / L_{x}\right) \sin ^{2}\right.$ $\left.\left(2 \pi y / L_{y}\right)\right]$. Initial random perturbations on the magnetic field of amplitude 0.00005 break symmetry so that secondary magnetic islands are ejected. The electron inertia is $m_{e}=m_{i} / 25$. For all simulations, the system is evolved until transient effects have subsided and a quasi-steady state is achieved. Data are averaged over an extended steady time.

Simulations are run with $v_{s}$ varied from 0 to 1.2. An overview of the results is shown in Fig. 3, in which the outof-plane current density $J_{z}$ in a small region around the $\mathrm{X}$-line is plotted with representative magnetic field lines drawn in white for $v_{s}=0.0,0.4,0.8$, and 1.2 with the flow to the left above and right below the current sheet. Several conclusions can be drawn from these plots. First, there is reconnection for $v_{S}<c_{A}$, but not for $v_{S}>c_{A}$. Further, the current
TABLE I. Measured quantities for Hall reconnection simulations. Blank entries correspond to values for which reliable measures are not attainable.

\begin{tabular}{lcccccccc}
\hline \hline$v_{s}$ & 0.0 & 0.2 & 0.4 & 0.6 & 0.7 & 0.8 & 0.9 & 1.0 \\
\hline$E$ & 0.069 & 0.069 & 0.055 & 0.045 & 0.038 & 0.025 & 0.008 & 0.013 \\
$B$ & 0.81 & 0.82 & 0.74 & 0.68 & 0.65 & 0.50 & & \\
$B_{e}$ & 0.42 & 0.42 & 0.37 & 0.33 & 0.28 & 0.23 & & \\
$v_{\text {out }}$ & 1.26 & 1.18 & 1.03 & 0.40 & & & & \\
$v_{e, \text { out }}$ & 1.64 & 1.69 & 1.33 & 1.12 & 0.75 & 0.69 & & \\
$\delta$ & 1.08 & 1.05 & 0.97 & 0.78 & & & & \\
$L_{L}$ & 24.4 & 17.6 & 17.9 & 1.9 & & & & \\
$\delta_{e}$ & 0.20 & 0.20 & 0.21 & 0.21 & 0.22 & 0.22 & & \\
$L_{e}$ & 1.90 & 1.92 & 1.69 & 1.49 & 0.89 & 0.86 & & \\
$\theta_{\text {tilt }}$ & 0.0 & 2.1 & 4.7 & 13.0 & 12.5 & 17.7 & 22.6 & 11.5 \\
$\theta_{\text {open }}$ & 9.6 & 9.3 & 11.0 & 12.0 & 18.4 & 20.8 & 27.8 & 25.8 \\
\hline \hline
\end{tabular}

sheets tilt in the direction in which the outflow and shear flow are anti-parallel, and the opening angle and tilt angle increase with $v_{s}$. Each of these phenomena were seen earlier in magnetohydrodynamics simulations with a localized resistivity, ${ }^{29-31,34}$ hybrid simulations, ${ }^{32}$ and particle-in-cell simulations. ${ }^{33}$ The present results show that the effects also occur in two-fluid simulations, so are likely robust signatures of reconnection with a shear flow.

To test the theory from Sec. II, physical parameters are extracted from the simulations. The results are compiled in Table I, and the techniques used to determine them and their scaling with $v_{s}$ are discussed in what follows. The reconnection rate $E$ and upstream magnetic fields $B$ and $B_{e}$ (at the ion and electron layer, respectively) were discussed in a previous publication, ${ }^{44}$ showing good agreement with Eqs. (6), (7), and (10).

The scaling of the outflow speed is tested both at the electron and ion layers. The electron outflow speed $v_{e, \text { out }}$ is measured as the maximum magnitude of the in-plane electron velocity. The length of the electron dissipation region $L_{e}$ is defined as the distance from the $\mathrm{X}$-line to where $v_{e, \text { out }}$ is measured. The results are plotted as the (blue) circles in Fig. 4. The data only go up to $v_{s}=0.8$ because the results are not reliable for higher $v_{s}$, where reconnection either does not occur or goes slowly in a very skewed geometry. The error bars are the standard deviations from averaging over a quasisteady time and often give reasonable estimates of the

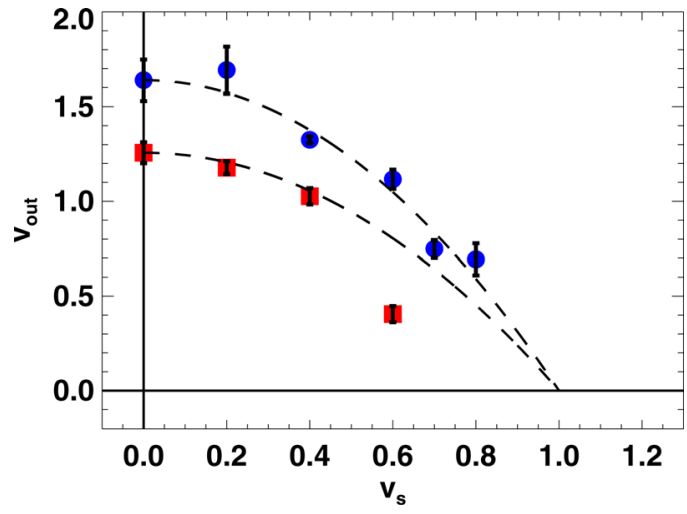

FIG. 4. (Color online) Ion (red boxes) and electron (blue circles) outflow speeds $v_{\text {out }}$ and $v_{e, \text { out }}$ as a function of shear flow speed $v_{s}$. The dashed lines are from Eqs. (8) and (11). 
uncertainty. The dashed line is $v_{e, \text { out }}=v_{e, \text { out } 0}\left(1-v_{s}^{2} / c_{A}^{2}\right)$, where $v_{e, \text { out }}$ is the electron outflow speed in the absence of a shear flow. This shows very good agreement with the prediction in Eq. (11) using the known result that $v_{e, \text { out } 0} \sim c_{A e}$.

The ion outflow speed $v_{\text {out }}$ is very difficult to measure due to the twisting of the current sheet. In fact, Ref. 25 showed using linear theory that the ion layer goes unstable to the Kelvin-Helmholtz instability while the electron layer continues to undergo reconnection for large enough shear flow speeds. The most reliable measure of $v_{\text {out }}$ identified for the present study is determined from the out-of-plane ion current $J_{i z}=n v_{i z}$. The ions are accelerated out of the plane only in the ion dissipation region, so $J_{i z}$ being non-zero is a good proxy for the dissipation region. The length of the ion dissipation region $L$ is defined as the location along a cut horizontally through the $\mathrm{X}$-line in which $J_{i z}$ is half its maximum value. Then, the ion outflow speed $v_{\text {out }}$ is defined as the maximum of the horizontal ion velocity $v_{i x}$ in a vertical cut at this location. This measure is only reliable for $v_{s} \leq 0.6$, which is comparable to the flow speed found in Ref. 25 at which the ion layer goes unstable to Kelvin-Helmholtz (see their Fig. 2).

The results for $v_{\text {out }}$ are plotted as (red) boxes in Fig. 4. The anticipated uncertainties for this measurement are far larger than the error bars determined by the standard deviation from the mean. The dashed line is $v_{\text {out }}=v_{\text {out }, 0}\left(1-v_{s}^{2} / c_{A}^{2}\right)$, where $v_{\text {out }, 0}$ is the outflow speed in the absence of shear flow. When using the known result that $v_{\text {out }, 0} \sim c_{A}$, the prediction from Eq. (8) shows good agreement for small $v_{s}$. Above $v_{s} \sim 0.6$, the prediction fails as the ion current sheet goes Kelvin-Helmholtz unstable. To illustrate that this instability is present, a plot of the plasma density zoomed near the X-line is plotted in Fig. 5 for the $v_{s}=0.8$ simulation, showing a characteristic roll, consistent with Ref. 25.

Next, the scaling of the shape of the dissipation region is addressed. The thickness $\delta_{e}$, of the electron dissipation region, is defined as the $e$-folding distance of the out-ofplane current $J_{z}$, in a cut in the direction of the inflow, which self-consistently tilts in simulations with a non-zero shear flow. For the ions, the thickness $\delta$ of the dissipation region is defined as the location in a vertical cut through the $\mathrm{X}$-line where the difference between the ion and electron inflows exceeds $15 \%$ of the maximum ion inflow speed, similar to the technique used in Ref. 46.

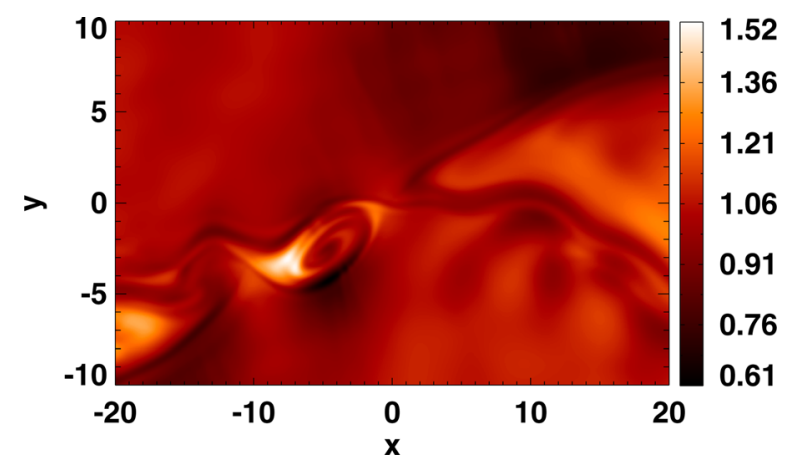

FIG. 5. (Color online) Plasma density $n$ zoomed in near the X-line for the $v_{s}=0.8$ simulation, showing a roll characteristic of the Kelvin-Helmholtz instability, consistent with Ref. 25.
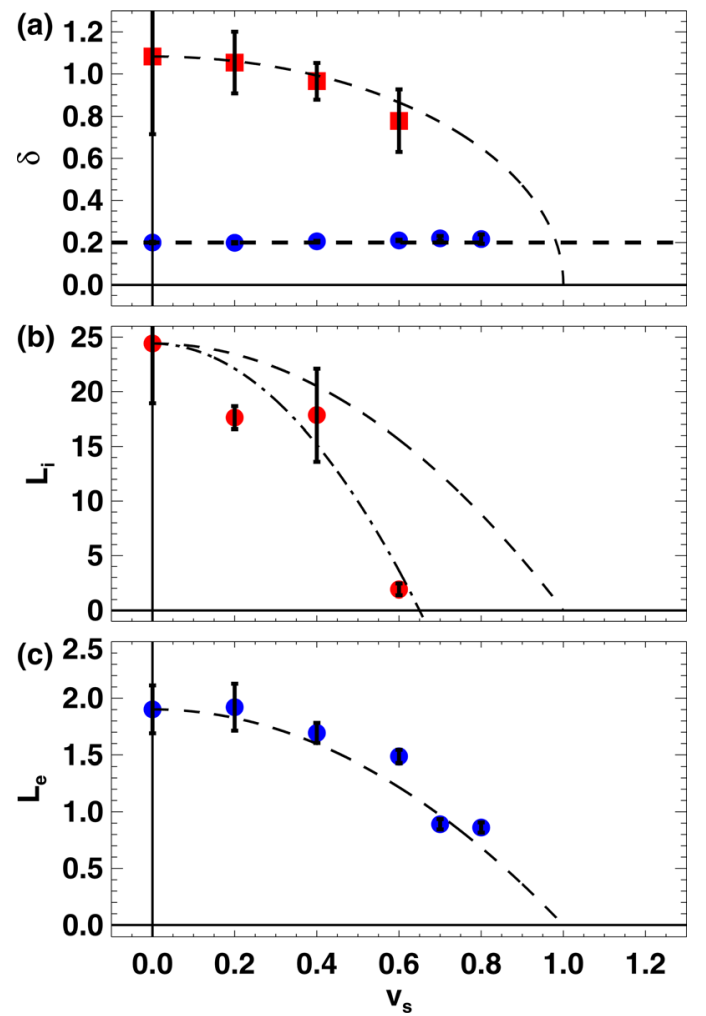

FIG. 6. (Color online) (a) Ion and electron thicknesses $\delta$ (red boxes) and $\delta_{e}$ (blue circles), (b) length $L$ of the ion dissipation region, and (c) length $L_{e}$ of the electron dissipation region as a function of shear flow speed $v_{s}$. Dashed lines show Eq. (3) for (a) and Eq. (9) for (b) and (c). The dot-dashed line in (b) is Eq. (15).

The results are plotted in Fig. 6. Panel (a) shows $\delta$ as the (red) boxes and $\delta_{e}$ as the (blue) circles. For the ions, the dashed line is $\delta_{i 0}\left(1-v_{s}^{2} / c_{A}^{2}\right)^{1 / 2}$, where $\delta_{i 0}$ is the thickness in the absence of shear flow. This is in good agreement with Eq. (3).

The electron layer thickness, on the other hand, is approximately the electron inertial length $d_{e}=c / \omega_{p e}=0.2$ independent of $v_{s}$, which disagrees with Eq. (12). It is possible that the electrons truly behave differently than the ions because they are at smaller scales and there is less of a shear flow at that scale, but it is also possible that this result is an artifact of the simulations. The grid in use is about $d_{e} / 4$, so it is questionable whether the grid scale dissipation can allow the electron layer to become thinner if it wanted to. Future simulations with higher resolution will be required to test the scaling of $\delta_{e}$.

The length $L$ of the ion layer is plotted in Fig. 6(b), again only up to $v_{s}=0.6$. The prediction from Eq. (9) is plotted as the dashed line. While there is definitely a trend where $L$ decreases with increasing $v_{s}$, the lack of quantitative agreement is not terribly surprising given the large uncertainty of measuring $L$. It is interesting to note that if one modifies the prediction to take into account the cutoff in $v_{s}$ at smaller ion flow speeds by postulating an expression of the form

$$
L \sim L_{0}\left(1-\frac{v_{s}^{2}}{\left(0.65 c_{A}\right)^{2}}\right),
$$

which is shown as the dotted-dashed line, one finds much better quantitative agreement. Whether this result is robust 


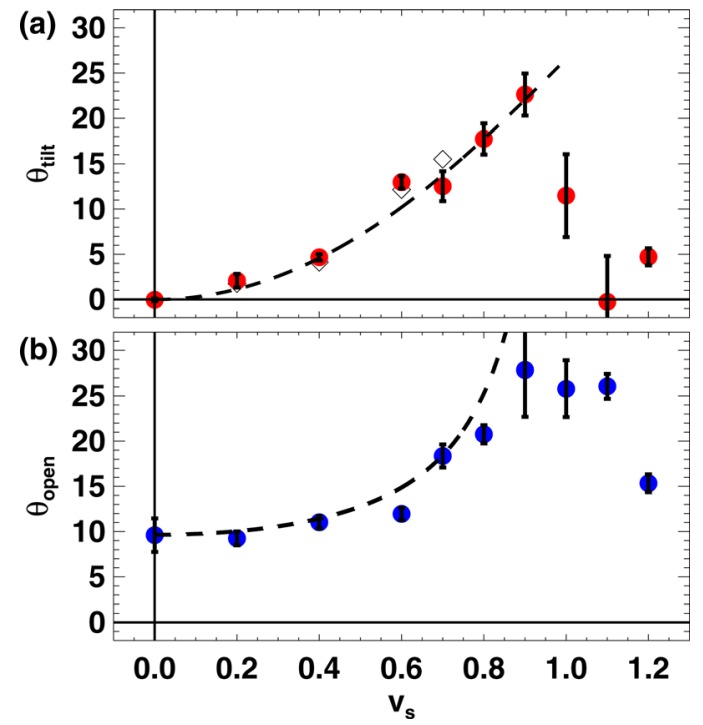

FIG. 7. (Color online) (a) Tilt angle $\theta_{\text {tilt }}$ and (b) opening angle $\theta_{\text {open }}$ as a function of shear flow speed $v_{s}$. The dashed lines are the predictions from Eqs. (14) and (17), respectively.

could be a topic of future study. The length $L_{e}$ of the electron layer is plotted in panel (c), with the predicted scaling from Eq. (13) plotted as the dashed line. The agreement is quite good.

Next, the scaling of the tilt angle $\theta_{\text {tilt }}$ is addressed. This angle is measured in two different ways. The first uses Hessian theory, which was used profitably recently in a study of reconnection sites in turbulence. ${ }^{47,48}$ The Hessian matrix $H$, at any location is defined as

$$
H=\left(\begin{array}{ll}
\frac{\partial^{2} \psi}{\partial x^{2}} & \frac{\partial^{2} \psi}{\partial x \partial y} \\
\frac{\partial^{2} \psi}{\partial x \partial y} & \frac{\partial^{2} \psi}{\partial y^{2}}
\end{array}\right)
$$

where $\psi$ is the flux function defined by $\mathbf{B}_{\perp}=-\hat{\mathbf{z}} \times \nabla \psi$ and $\mathbf{B}_{\perp}$ is the in-plane magnetic field. The eigenvectors of $H$ at the X-line (a saddle-point in $\psi$ ) provide the principal axes of the inflow and outflow locally. From this, $\theta_{\text {tilt }}$ is found as the angle the eigenvector corresponding to the outflow direction (the smaller eigenvalue) makes with the horizontal. The results are plotted as the (red) circles in Fig. 7(a). Alternately, the location where $v_{e, \text { out }}$ is measured (that defines $L_{e}$ ) should also be a good measure of the tilt angle. For simulations in which a reliable value can be ascertained $\left(v_{s} \leq 0.7\right)$, the results are plotted as the open diamonds in Fig. 7(a). In most cases, the difference between the two measurements is small enough that the points overlap, which gives confidence that $\theta_{\text {tilt }}$ is being determined appropriately. The dashed line in Fig. 7(a) gives the prediction from Eq. (14), showing excellent agreement. Thus, the conclusion is that the dynamic pressure of the shear flow on the newly reconnected field lines causes the outflow jet to tilt.

Finally, the opening angle $\theta_{\text {open }}$ of the magnetic field lines in the outflow region is also determined from the eigenvalues of the Hessian matrix at the X-line, being the inverse tangent of the ratio of the eigenvectors. This is plotted in Fig. 7(b). To understand the dependence, note that $\tan \left(\theta_{\text {open }}\right) \sim \delta_{e} / L_{e}$. The results of Figs. 6(a) and (c) suggest a scaling of

$$
\tan \left(\theta_{\text {open }}\right) \sim \frac{\tan \left(\theta_{\text {open }, 0}\right)}{\left(1-v_{s}^{2} / c_{A}^{2}\right)},
$$

where $\theta_{\text {open }, 0}$ is the opening angle in the absence of shear flow. This expression is shown as the dashed line, showing good agreement for systems that reconnect $\left(v_{s}<1\right)$. Note, however, that this angle is determined by electron physics, which is not necessarily resolved well enough in the present simulations, so higher resolution simulations would be necessary to check this result.

To summarize the results of the Hall reconnection simulation study, the predictions from Sec. II agree rather well with the numerical results.

\section{A. Sweet-Parker reconnection with shear flow}

For many applications of reconnection with a shear flow, the collisionless regime of Hall reconnection is most relevant. However, for completeness, interesting results on differences between Hall and collisional (Sweet-Parker) reconnection in the presence of a shear flow are reported. The differences are somewhat surprising given that the scaling of reconnection with an asymmetry in field strength and density are similar for both types of reconnection. ${ }^{45,49-52}$

The effect of shear flow on Sweet-Parker reconnection is studied with resistive-MHD simulations again using the F3D $\operatorname{code}^{46}$ (without the Hall term). The normalization is slightly different than in the two-fluid simulations as there is no ion inertial scale in MHD; instead, lengths are normalized to an arbitrary length $l^{\prime}$ and times to the Alfvén time $l^{\prime} / c_{A}^{\prime}$. Otherwise, all normalizations are the same as in the two-fluid simulations.

The simulations are similar to the two-fluid ones, with a two-dimensional periodic domain of size $204.8 \times 102.4$ with a grid scale of 0.05 . The fourth order dissipation has coefficient $2 \times 10^{-5}$. The electron mass is now zero, and a constant and uniform resistivity of $\eta=0.01$ is employed. This value is chosen large enough to prevent spontaneous production of secondary islands. ${ }^{53-60}$ Otherwise, all other parameters and initializations are as in the two-fluid simulations described previously. The shear flow speed $v_{s}$ is varied and reconnection parameters are measured; their values are given in Table II.

As with Hall reconnection, one expects increasing shear flow to slow the outflow speed $v_{\text {out }}$ and reduce the reconnection rate $E$. This is borne out in the simulations, as shown in Fig. 8. Panel (a) contains $E$, measured as the time rate of change in magnetic flux between the $\mathrm{X}$-line and the O-line,

TABLE II. Measured parameters from resistive-MHD simulations of reconnection with a shear flow.

\begin{tabular}{lcccccc}
\hline \hline$v_{s}$ & 0.0 & 0.1 & 0.2 & 0.3 & 0.4 & 0.5 \\
\hline$E$ & 0.015 & 0.014 & 0.013 & 0.012 & 0.011 & 0.010 \\
$v_{\text {out }}$ & 0.97 & 0.90 & 0.86 & 0.72 & 0.80 & 0.80 \\
$\delta$ & 0.70 & 0.71 & 0.74 & 0.76 & 0.82 & 0.86 \\
$L$ & 41.4 & 38.3 & 36.8 & 33.5 & 32.2 & 22.7 \\
\hline \hline
\end{tabular}



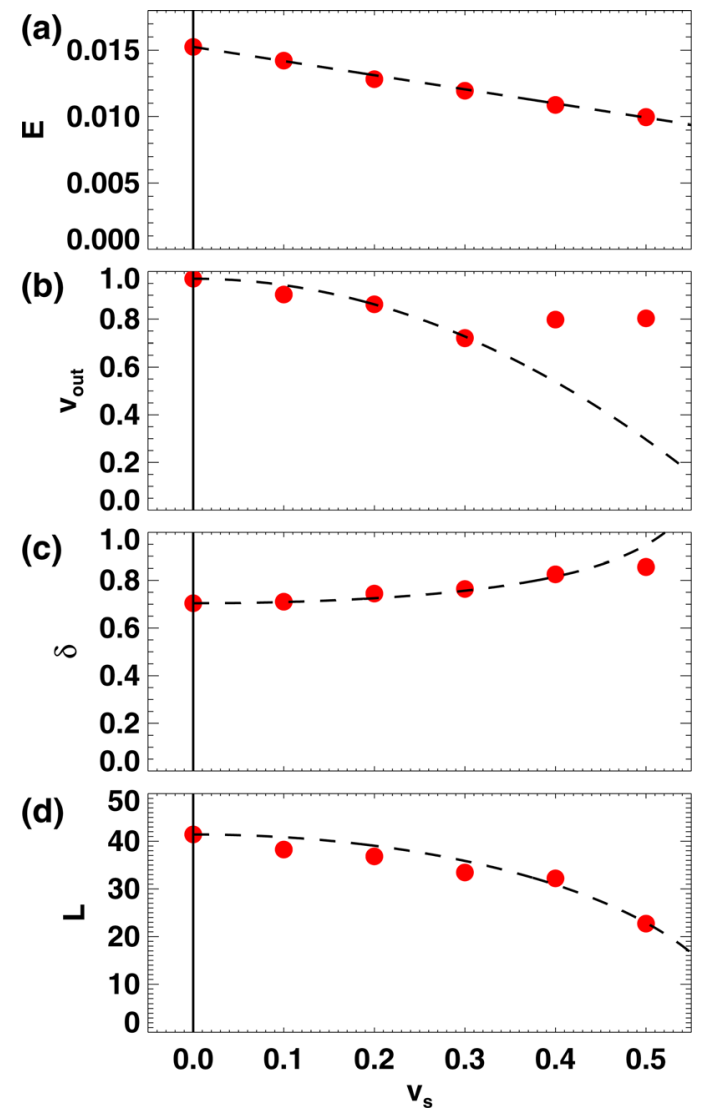

FIG. 8. (Color online) (a) Reconnection rate $E$, (b) outflow speed $v_{\text {out }}$, (c) dissipation region thickness $\delta$, (d) and dissipation region length $L$ as a function of shear flow speed $v_{s}$ for the collisional reconnection simulations.

which decreases with increasing $v_{s}$. An interesting difference is that a shear flow of $v_{s}=0.6$ is sufficient to suppress reconnection rather than 1 for Hall reconnection (the present study) or resistive-MHD with a localized resistivity. ${ }^{26}$ The dashed line is a linear fit to the data; it does not represent a theoretical prediction, which is outside the scope of the present study.

The outflow speed $v_{\text {out }}$ is measured as the average of the maximum horizontal flow speed in the two outflow directions, and is plotted in Fig. 8(b). A decrease in $v_{\text {out }}$ with $v_{s}$ is observed as expected. For reference,

$$
v_{\text {out }}=v_{\text {out }, 0}\left(1-\frac{v_{s}^{2}}{\left(0.6 c_{A}\right)^{2}}\right)
$$

is plotted as the dashed line, corresponding to Eq. (8), corrected for reconnection shutting off at 0.6 by analogy with Eq. (15). There is some agreement for small $v_{s}$. The increase in $v_{\text {out }}$ for large $v_{s}$ may be physical, but is also likely affected by a large uncertainty. In particular, the $v_{s}=0.3$ run had more than one time that could have been called a steadystate, so its $v_{\text {out }}$ could be somewhat different. More work would be necessary to better understand the scaling, but that is beyond the scope of the present work.

A second interesting departure from Hall reconnection is the scaling of the half-thickness $\delta$ with $v_{s}$. In Hall reconnection, the thickness decreases with $v_{s}$, as described by Eq. (3). However, the layer broadens for Sweet-Parker reconnection, as shown in Fig. 8(c) in which $\delta$ is measured as the $e$-folding distance of the out-of-plane current $J_{z}$ in the direction of the inflow, which tilts self-consistently for simulations with a non-zero shear flow. The reason is very simple; in the Sweet-Parker model, a steady-state occurs when diffusion balances the convection of magnetic flux into the dissipation region. This requires ${ }^{1}$

$$
v_{i n} \sim \frac{\eta c^{2}}{4 \pi \delta},
$$

where $v_{\text {in }}$ is the inflow speed. Since $E \propto v_{\text {in }}$, a decrease in reconnection rate is accompanied by a broadening of the dissipation region, as is observed in the simulations. The dashed line is $\delta_{0} /\left[1-v_{s}^{2} /\left(0.6 c_{A}\right)^{2}\right]^{1 / 4}$, which is plotted to guide the eye and is not a prediction.

Finally, the length $L$ is measured as the straight line distance from the X-line to the point at which $v_{\text {out }}$ is measured and is plotted in Fig. 8(d). Clearly, $L$ decreases with $v_{s}$. For reference,

$$
L=L_{0} \sqrt{1-\frac{v_{s}^{2}}{\left(0.6 c_{A}\right)^{2}}}
$$

is plotted as the dashed line, which generalizes Eq. (4). The fit is pretty good, which suggests that the upstream magnetic field does not change much when the layer broadens, which is very reasonable.

It is important to note that the intention of Fig. 8 is not to establish scaling laws for the Sweet-Parker system but rather solely to show trends. A more complete analysis to establish particular scaling laws is required if a quantitative prediction for this system is desired.

\section{SUMMARY AND CONCLUSIONS}

This paper addresses the scaling of collisionless (Hall) magnetic reconnection in the presence of a shear flow. Specifically, the flow is initially parallel to the reconnecting magnetic field and has the same speed on either side of the dissipation region. A theoretical model of the effect of shear flow is presented. The crux is that the bulk flow releases some of the tension in newly reconnected magnetic field lines, which accelerates the outflow less efficiently and causes a slowing down of the reconnection process. Scaling arguments for the outflow speed, reconnection rate, and dissipation region thickness and length are presented, revealing that each quantity decreases with increasing shear flow speed. These predictions are qualitatively consistent with previous simulation results. ${ }^{26,30-34}$ The ion layer is inferred to be Kelvin-Helmholtz unstable at shear flows above $0.6 c_{A}$, consistent with linear theory results from Ref. 25. Further, a previous observation that the shear flow causes the dissipation region to tilt in the direction in which the outflow and shear flow are anti-aligned ${ }^{26}$ is explained physically as due to the dynamic pressure of the shear flow on newly reconnected magnetic field lines. A scaling argument predicting the angle is presented. All of the scaling relations are tested using two-dimensional two-fluid numerical simulations of anti-parallel magnetic reconnection. The numerical results 
agree with the predictions rather well. Finally, some important differences between collisionless and collisional (SweetParker) reconnection with a shear flow are pointed out.

The predictions derived here, or relevant extensions of them, may be very useful for quantitative analyses of magnetic reconnection in the presence of a shear flow, which occurs at the high latitudes of the magnetopause due to the solar wind, as well as tokamak applications, and potentially reconnection in the solar wind and in reconnection in turbulent plasmas. ${ }^{47}$ It is doubtful the effects of shear flow are important in the solar corona because the solar wind speed is much smaller than the Alfvén speed. Quantitative comparisons in appropriate settings would be potentially very interesting.

There are a few important limitations of the present simulations. One is that physics below the electron inertial scale is not captured well by the two-fluid model, and the grid scale resolution is only marginally below electron inertial scales. Therefore, it is not clear whether the changes to the electron layer with shear flow observed in this study are physical or numerical. Future work, perhaps involving particle-in-cell simulations, will likely be necessary to fully understand effects at the electron layer. Also, the treatment of Sweet-Parker reconnection with a shear flow given here is incomplete and will require future work if scaling results are needed.

Additional studies should also involve relaxing the simplifying assumptions of the model both theoretically and numerically, such as two-dimensionality, anti-parallel fields, asymmetries in the shear flow speed, and asymmetries in the magnetic field and densities. Such studies will be necessary to make the results applicable to most physical systems and will be the subject of future work.

\section{ACKNOWLEDGMENTS}

The author thanks M. A. Shay for some computational resources and T. D. Phan for helpful conversations. Support by NSF Grant No. AGS-0953463 and NASA Grant No. NNX10AN08A is gratefully acknowledged. This research used resources of the National Energy Research Scientific Computing Center, which is supported by the Office of Science of the U.S. Department of Energy under Contract No. DE-AC02-05CH11231.

${ }^{1}$ E. N. Parker, J. Geophys. Res. 62, 509, doi:10.1029/JZ062i004p00509 (1957).

${ }^{2}$ H. E. Petschek, in AAS/NASA Symposium on the Physics of Solar Flares, edited by W. N. Ness (NASA, Washington, DC, 1964), p. 425.

${ }^{3}$ R. H. Levy, H. E. Petschek, and G. L. Siscoe, AIAA J. 2, 2065 (1964).

${ }^{4}$ H. E. Petschek and R. M. Thorne, Astrophys. J. 147, 1157 (1967).

${ }^{5}$ D. I. Pontin, Adv. Space Res. 47, 1508 (2011).

${ }^{6}$ R. J. La Haye, D. P. Brennan, R. J. Buttery, and S. P. Gerhardt, Phys. Plasmas 17, 056110 (2010).

${ }^{7}$ J. T. Gosling, M. F. Thomsen, and S. J. Bame, J. Geophys. Res. 91, 3029, doi:10.1029/JA091iA03p03029 (1986).

${ }^{8}$ J. T. Gosling, M. F. Thomsen, S. J. Bame, and R. C. Elphic, J. Geophys. Res. 96, 14,097, doi:10.1029/91JA01139 (1991).

${ }^{9}$ J. T. Gosling, M. F. Thomsen, G. Le, and C. T. Russell, J. Geophys. Res. 101, 24,765, doi:10.1029/96JA02254 (1996).

${ }^{10}$ R. L. Kessel, S.-H. Chen, J. L. Green, S. F. Fung, S. A. Boardsen, L. C. Tan, T. E. Eastman, J. D. Craven, and L. A. Frank, Geophys. Res. Lett. 23, 583, doi:10.1029/95GL03083 (1996).
${ }^{11}$ T. G. Onsager, J. D. Scudder, M. Lockwood, and C. T. Russell, J. Geophys. Res. 106, 25,467, doi:10.1029/2000JA000444 (2001).

${ }^{12}$ L. A. Avanov, V. N. Smirnov, J. H. Waite Jr., S. A. Fuselier, and O. L. Vaisberg, J. Geophys. Res. 106, 29,491, doi:10.1029/2000JA000460 (2001).

${ }^{13}$ A. Federov, E. Dubinin, P. Song, A. Skalsky, and E. Budnik, J. Geophys. Res. 106, 25,419, doi:10.1029/2000JA900104 (2001).

${ }^{14}$ T. D. Phan, B. U. Ö. Sonnerup, and R. P. Lin, J. Geophys. Res. 106, 25,489, doi:10.1029/2001JA900054 (2001).

${ }^{15}$ T. Phan, H. U. Frey, S. Frey, L. Peticolas, S. Fuselier, C. Carlson, H. Rème, J.-M. Bosqued, A. Balogh, M. Dunlop, L. Kistler, C. Mouikis, I. Dandouras, J. A. Sauvard, S. Mende, J. McFadden, G. Parks, E. Moebius, B. Klecker, G. Paschmann, M. Fujimoto, S. Petrinec, M. F. Martucci, A. Korth, and R. Lundin, Geophys. Res. Lett. 30, 1509, doi:10.1029/2003GL016885 (2003).

${ }^{16}$ T. D. Phan, H. Hasegawa, M. Fujimoto, M. Oieroset, T. Mukai, R. P. Lin, and W. Paterson, Geophys. Res. Lett. 33, L09104, doi:10.1029/ 2006GL025756 (2006).

${ }^{17}$ T. D. Phan, G. Paschmann, C. Twitty, F. S. Mozer, J. T. Gosling, J. P. Eastwood, M. Øieroset, H. Rème, and E. A. Lucek, Geophys. Res. Lett. 34, L14104, doi:10.1029/2007GL030343 (2007).

${ }^{18}$ S. Eriksson, J. T. Gosling, T. D. Phan, L. M. Blush, K. D. C. Simunac, D. Krauss-Varban, A. Szabo, J. G. Luhmann, C. T. Russell, A. B. Galvin, and M. H. Acuña, J. Geophys. Res. 114, A07103, doi:10.1029/2008JA013990 (2009).

${ }^{19}$ C. Twitty, T. D. Phan, G. Paschmann, B. Lavraud, H. Rème, and M. Dunlop, Geophys. Res. Lett. 31, L19808, doi:10.5194/angeo-22-2355-2004 (2004).

${ }^{20}$ M. Longmore, S. J. Schwartz, J. Geach, B. M. A. Cooling, I. Dandouras, E. A. Lucek, and A. N. Fazakerley, Ann. Geophys. 23, 3351, doi:10.5194/ angeo-23-3351-2005 (2005).

${ }^{21}$ H. G. Mitchell, Jr. and J. R. Kan, J. Plasma Phys. 20, 31 (1978).

${ }^{22}$ M. F. Heyn, H. K. Biernat, R. P. Rijnbeek, and V. S. Semenov, J. Plasma Phys. 40, 235 (1988)

${ }^{23}$ H. K. Biernat, M. F. Heyn, R. P. Rijnbeek, V. S. Semenov, and C. J. Farrugia, J. Geophys. Res. 94, 287, doi:10.1029/JA094iA01p00287 (1989).

${ }^{24}$ X. L. Chen and P. J. Morrison, Phys. Fluids B 2, 495 (1990).

${ }^{25}$ L. Chacón, D. A. Knoll, and J. M. Finn, Phys. Lett. A 308, 187 (2003).

${ }^{26}$ A. L. La Belle-Hamer, A. Otto, and L. C. Lee, Phys. Plasmas 1, 706 (1994).

${ }^{27}$ M. Swisdak, J. F. Drake, M. A. Shay, and B. N. Rogers, J. Geophys. Res. 108, 1218, doi:10.1029/2002JA009726 (2003).

${ }^{28}$ T. D. Phan, J. T. Gosling, G. Paschmann, C. Pasma, J. F. Drake, M. Øieroset, D. Larson, R. P. Lin, and M. S. Davis, Astrophys. J. Lett. 719, L199 (2010).

${ }^{29}$ A. L. La Belle-Hamer, A. Otto, and L. C. Lee, J. Geophys. Res. 100, 11,875, doi:10.1029/94JA00969 (1995).

${ }^{30}$ Q. Chen, A. Otto, and L. C. Lee, J. Geophys. Res. 102, 151, doi:10.1029/ 97JD01107 (1997).

${ }^{31}$ J. H. Li and Z. W. Ma, J. Geophys. Res. 115, A09216, doi:10.1029/ 2010JA015315 (2010).

${ }^{32}$ H. Xie and Y. Lin, J. Geophys. Res. 105, 25171, doi:10.1029/ 2000JA000143 (2000).

${ }^{33}$ K. Nishimura, S. P. Gary, H. Li, and S. Colgate, Phys. Plasmas 10, 347 (2003).

${ }^{34}$ Q. Q. Shi, Z. Y. Pu, H. Zhang, S. Y. Fu, C. J. Xiao, Q.-G. Zong, T. A Fritz, and Z. X. Liu, Surv. Geophys. 26, 369 (2005).

${ }^{35}$ V. Roytershteyn and W. Daughton, Phys. Plasmas 15, 082901 (2008).

${ }^{36}$ K. G. Tanaka, M. Fujimoto, and I. Shinohara, Int. J. Geophys. 2010, 202583 (2010).

${ }^{37}$ H. C. Ku and D. G. Sibeck, J. Geophys. Res. 103, 6693, doi:10.1029/ 97JA03688 (1998).

${ }^{38}$ G. Einaudi, P. Boncinelli, R. B. Dahlburg, and J. T. Karpen, J. Geophys. Res. 104, 521, doi:10.1029/98JA02394 (1999).

${ }^{39}$ G. Einaudi, S. Chibbaro, R. B. Dahlburg, and M. Velli, Astrophys. J. 547, 1167 (2001).

${ }^{40}$ L. Bettarini, S. Landi, F. A. Rappazzo, M. Velli, and M. Opher, Astron. Astrophys. 452, 321 (2006).

${ }^{41}$ L. Bettarini, S. Landi, M. Velli, and P. Londrillo, Phys. Plasmas 16, 062302 (2009).

${ }^{42}$ L. Ni, K. Germaschewski, Y.-M. Huang, B. P. Sullivan, H. Yang, and A. Bhattacharjee, Phys. Plasmas 17, 052109 (2010).

${ }^{43}$ J. E. Borovsky, J. Geophys. Res. 113, A08228, doi:10.1029/ 2007JA012646 (2008).

${ }^{44}$ P. A. Cassak and A. Otto, Phys. Plasmas 18, (2011).

${ }^{45}$ P. A. Cassak and M. A. Shay, Phys. Plasmas 16, 055704 (2009).

${ }^{46}$ M. A. Shay, J. F. Drake, M. Swisdak, and B. N. Rogers, Phys. Plasmas 11, 2199 (2004) 
${ }^{47}$ S. Servidio, W. H. Matthaeus, M. A. Shay, P. A. Cassak, and P. Dmitruk, Phys. Rev. Lett. 102, 115003 (2009).

${ }^{48}$ S. Servidio, W. H. Matthaeus, M. A. Shay, P. Dmitruk, P. A. Cassak, and M. Wan, Phys. Plasmas 17, 032315 (2010).

${ }^{49}$ P. A. Cassak and M. A. Shay, Phys. Plasmas 14, 102114 (2007).

${ }^{50}$ P. A. Cassak and M. A. Shay, Geophys. Res. Lett. 35, L19102, doi:10.1029/2008GL035268 (2008).

${ }^{51}$ K. Malakit, M. A. Shay, P. A. Cassak, and C. Bard, J. Geophys. Res. 115, A10223, doi:10.1029/2010JA015452 (2010).

${ }^{52}$ N. A. Murphy, C. R. Sovinec, and P. A. Cassak, J. Geophys. Res. 115, A09206, doi:10.1029/2009JA015183 (2010).

${ }^{53}$ D. Biskamp, Phys. Fluids 29, 1520 (1986).

${ }^{54}$ W. Daughton, V. Roytershteyn, B. J. Albright, H. Karimabadi, L. Yin, and K. J. Bowers, Phys. Rev. Lett. 103, 065004 (2009).

${ }^{55}$ A. Bhattacharjee, Y.-M. Huang, H. Yang, and B. Rogers, Phys. Plasmas 16, 112102 (2009).
${ }^{56}$ P. A. Cassak, M. A. Shay, and J. F. Drake, Phys. Plasmas 16, 102702 (2009).

${ }^{57}$ P. A. Cassak and J. F. Drake, Astrophys. J. Lett. 707, L158 (2009).

${ }^{58}$ Y.-M. Huang and A. Bhattacharjee, Phys. Plasmas 17, 062104 (2010).

${ }^{59}$ L. S. Shepherd and P. A. Cassak, Phys. Rev. Lett. 105, 015004 (2010).

${ }^{60}$ D. A. Uzdensky, N. F. Loureiro, and A. A. Schkochihin, Phys. Rev. Lett. 105, 235002 (2010).

${ }^{61}$ B. Lavraud, T. D. Phan, M. W. Dunlop, M. G. G. T. Taylor, P.J. Cargill, J.-M. Bosqued, I. Dandouras, H. Rème, J.-A. Sauvaud, C. P. Escoubet, A. Balogh, and A. Fazakerley, Ann. Geophys. 22, 3039, doi:10.5194/angeo22-3039-2004 (2004).

${ }^{62}$ B. Lavraud, A. Fedorov, E. Budnik, M. F. Thomsen, A. Grigoriev, P. J. Cargill, M. W. Dunlop, H. Rème, I. Dandouras, and A. Balogh, J. Geophys. Res., 110, A02209, doi:10.1029/2004JA010804 (2005).

${ }^{63}$ H. Che, M. V. Goldman, and D. L. Newman, Phys. Plasmas, 18, 052109 (2011). 